\title{
Characteristics of patients from the Polish Registry of Acute Coronary Syndromes during the COVID-19 pandemic: the first report
}

\author{
Michał Hawranek', Marek Grygier², Kamil Bujak', Stanisław Bartuś3, Marek Gierlotka4, \\ Wojciech Wojakowski ${ }^{5}$, Jacek Legutko ${ }^{6,7}$, Maciej Lesiak² ${ }^{2}$ Piotr Pączek ${ }^{8}$, Andrzej Kleinrok ${ }^{9}$, Krzysztof \\ Milewski ${ }^{10}$, Jacek Kubica ${ }^{11}$, Mateusz Tajstra ${ }^{1}$, Dariusz Dudek ${ }^{12,13}$, Adam Witkowski ${ }^{14}$, Mariusz Gąsior ${ }^{1}$ \\ 1 3rd Department of Cardiology, Faculty of Medical Sciences in Zabrze, Medical University of Silesia, Katowice, Poland \\ 2 1st Department of Cardiology, Poznan University of Medical Sciences, Poznań, Poland \\ 3 2nd Deptartment of Cardiology and Cardiovascular Interventions, Jagiellonian University Medical College, Kraków, Poland \\ 4 Department of Cardiology, University Hospital in Opole, University of Opole, Opole, Poland \\ 5 Department of Cardiology and Structural Heart Diseases, 3rd Division of Cardiology, Medical University of Silesia, Katowice, Poland \\ 6 Department of Interventional Cardiology, Institute of Cardiology, Jagiellonian University Medical College, Kraków, Poland \\ 7 Clinical Department of Interventional Cardiology, John Paul II Hospital, Kraków, Poland \\ 8 Department of Cardiology, Public Clinical Hospital, Sosnowiec, Poland \\ 9 Department of Cardiology, The Pope John Paul II Province Hospital of Zamość, Zamość, Poland \\ 10 Cardiology and Cardiac Surgery Center, Center for Cardiovascular Research and Development, American Heart of Poland, Bielsko-Biała, Poland \\ 11 Cardiovascular Institute, Ludwik Rydygier Collegium Medicum, Nicolaus Copernicus University, Bydgoszcz, Poland \\ 12 Institute of Cardiology, Jagiellonian University Medical College, Kraków, Poland \\ 13 Maria Cecilia Hospital, GVM Care \& Research, Cotignola, Italy \\ 14 Department of Interventional Cardiology and Angiology, Institute of Cardiology in Warsaw, Warsaw, Poland
}

Correspondence to: Michał Hawranek, MD, PhD, 3rd Department of Cardiology, Silesian Center for Heart Diseases, Faculty of Medicine in Zabrze, Medical University of Silesia, ul. Skłodowskiej-Curie 9 , 41-800 Zabrze, Poland, phone: +48323733860 , email: mhawranek@poczta.fm Received: October 26, 2020. Revision accepted: January 7, 2021. Published online: January 18, 2021. Kardiol Pol. 2021; 79 (2): 192-195 doi:10.33963/KP.15756 Copyright by the Author(s), 2021
Introduction The coronavirus disease 2019 (COVID-19) pandemic has affected the entire global healthcare system. According to reports from different countries, the number of patients with myocardial infarction (MI) treated in 2020 has decreased by $40 \%$ to $50 \%$ as compared with the corresponding period in 2019. ${ }^{1-7}$ This refers both to patients with ST-segment elevation myocardial infarction (STEMI) and with non-ST-segment elevation myocardial infarction (NSTEMI). ${ }^{2,5,6}$ A similar situation occurred in Poland, even though cardiology centers were prepared for activity and provided appropriate logistics. ${ }^{6,7}$

Except for data on the number of patients, so far, there has been little information on the clinical characteristics of MI patients referred for invasive diagnostics during the COVID-19 pandemic. An Italian registry showed that patients treated at cardiology centers during the epidemic were older than those treated in 2019. Additionally, there were fewer women among patients treated for MI. ${ }^{5}$

Based on the data from the Polish Registry of Acute Coronary Syndromes (PL-ACS) collected during the COVID-19 era and the corresponding period in 2019, we compared treatment logistics and clinical characteristics of patients with acute coronary syndromes (ACS) treated in these 2 time intervals.

Methods The Polish Registry of Acute Coronary Syndromes (PL-ACS) is a national, prospective registry which, since 2003 , has been collecting data on acute coronary syndromes, including the incidence rate, patient clinical characteristics, therapeutic procedures as well as in-hospital and long-term outcomes. Currently, the registry data are entered by the attending physician via an online form. In 2019, the median (interquartile range [IQR]) time from admission of an ACS patient to reporting the patient file in the computer system was 32.5 (981) days. By the end of 2019, data on 749821 patients with ACS were gathered in the PL-ACS. More information on the registry was provided previously. ${ }^{8}$

Patients with a diagnosis of STEMI or NSTEMI hospitalized during the lockdown period of 
TABLE 1 Characteristics of patients diagnosed with acute myocardial infarction before and during the coronavirus disease 2019 pandemic

Variable

Age, y

Male sex

Mode of admission

March-May $2019(n=7844)^{a} \quad$ March-May $2020(n=3457)^{\mathrm{b}} \quad P$ value

$68(61-76)$

$5133(65.44)$

$67(60-75)$ $<0.01$

0.36 $<0.001$

\begin{tabular}{ll} 
Transfer from another hospital & $3157(40.42)$ \\
\hline Admission from home (through & $3236(41.43)$
\end{tabular}
the EMS)

\begin{tabular}{lll}
\hline Self-admission to hospital & $652(8.35)$ & $257(7.47)$ \\
\hline $\begin{array}{l}\text { Referred by the general } \\
\text { practitioner }\end{array}$ & $503(6.44)$ & $190(5.52)$ \\
\hline
\end{tabular}

\section{Referred by the specialist} outpatient clinic

\begin{tabular}{|c|c|c|c|c|}
\hline \multirow[t]{4}{*}{ Killip-Kimball classification } & $\mathrm{I}$ & $6312(81.57)$ & $2807(81.79)$ & \multirow[t]{4}{*}{0.73} \\
\hline & II & $983(12.7)$ & $417(12.15)$ & \\
\hline & III & $228(2.95)$ & $103(3)$ & \\
\hline & IV & $215(2.78)$ & $105(3.06)$ & \\
\hline \multirow[t]{2}{*}{ Diagnosis } & STEMI & $2828(36.05)$ & $1308(37.83)$ & \multirow[t]{2}{*}{0.07} \\
\hline & NSTEMI & $5017(63.95)$ & $2150(62.17)$ & \\
\hline \multicolumn{2}{|c|}{ Sudden cardiac arrest before admission } & $213(2.75)$ & $118(3.43)$ & 0.049 \\
\hline \multirow{4}{*}{$\begin{array}{l}\text { Time from onset of pain to } \\
\text { hospital admission }\end{array}$} & STEMI (direct admission) & $180(101-400)$ & $180(100-414)$ & 0.73 \\
\hline & $\begin{array}{l}\text { STEMI (transfer from another } \\
\text { hospital) }\end{array}$ & $316(171-720)$ & $290(146-697)$ & 0.14 \\
\hline & NSTEMI (direct admission) & $409(173-1285)$ & $390(173-1510)$ & 0.78 \\
\hline & $\begin{array}{l}\text { NSTEMI (transfer from another } \\
\text { hospital) }\end{array}$ & $609(298-1560)$ & $660(350-1589)$ & 0.09 \\
\hline \multirow{2}{*}{$\begin{array}{l}\text { Time from admission to } \\
\text { angiography, min }\end{array}$} & STEMI & $25(11-59)$ & $21(10-50.5)$ & $<0.001$ \\
\hline & NSTEMI & $229(61-839)$ & $232.5(68-897)$ & 0.32 \\
\hline \multicolumn{2}{|c|}{ Left ventricular ejection fraction, $\%$} & $50(40-55)$ & $48(40-55)$ & 0.16 \\
\hline \multicolumn{2}{|c|}{ Multivessel coronary artery disease } & $2994(44.12)$ & $1270(42.42)$ & 0.12 \\
\hline \multicolumn{2}{|c|}{ Percutaneous coronary interventions } & $6345(81.40)$ & $2839(82.22)$ & 0.30 \\
\hline \multirow[t]{4}{*}{ CABG } & Yes, during hospitalization & $15(0.19)$ & $7(0.21)$ & \multirow[t]{4}{*}{$<0.001$} \\
\hline & No, but referred for CABG & $234(3.04)$ & $119(3.49)$ & \\
\hline & No, but planned after discharge & $296(3.85)$ & $71(2.08)$ & \\
\hline & Not planned & $7148(92.92)$ & $3215(94.23)$ & \\
\hline \multicolumn{2}{|l|}{ Intra-aortic balloon pump } & $31(0.4)$ & $8(0.23)$ & 0.16 \\
\hline \multicolumn{2}{|l|}{ Cardiogenic shock } & $167(2.18)$ & $83(2.45)$ & 0.39 \\
\hline \multicolumn{2}{|l|}{ Length of hospitalization, $d$} & $5(3-7)$ & $5(3-7)$ & 0.56 \\
\hline
\end{tabular}

Data are presented as median (interquartile range) for continuous variables and number (percentage) of patients for categorical variables.

a Period from March 3, 2019 to May 31, 2019

b Period from March 1, 2020 to May 31, 2020

Abbreviations: CABG, coronary artery bypass grafting; EMS, Emergency Medical Services; NSTEMI, non-ST-segment elevation myocardial infarction; STEMI, ST-segment elevation myocardial infarction

the COVID-19 pandemic (March 1, 2020-May 31, 2020) were included in the present analysis. The control group comprised patients with a diagnosed MI hospitalized during the corresponding period in 2019 (March 1, 2019-May 31, 2019).
The analysis included demographic data, clinical characteristics, and hospital procedures reported to PL-ACS by May 1, 2020. The approval of an ethics committee was not required for this analysis. 
Statistical analysis The distribution of continuous variables was nonnormal based on the Shapiro-Wilk test; therefore, they were presented as medians and IQR and compared with the Mann-Whitney U test. Qualitative variables were presented as number and percentage of patients and compared with the $\mathrm{X}^{2}$ test. The $P$ value of less than 0.05 was considered significant. Statistical analysis was performed using the Statistica software, version 13.3 (TIBCO Software Inc., Palo Alto, California, United States).

Results and discussion Data on treatment logistics and clinical characteristics of the MI patients are presented in TABLE1. In comparison with the control group, patients treated in 2020 were slightly younger (median [IQR] age, 68 [61-76] years vs 67 [60-75] years, respectively), and more often experienced sudden cardiac arrest (SCA) prior to hospital admission ( $2.75 \%$ vs $3.43 \%$, respectively). There was also a trend toward a higher rate of STEMI during the COVID-19 period (36.05\% in 2019 vs $37.83 \%$ in 2020 ).

There is paucity of clinical data on MI patients treated during the COVID-19 pandemic. In an Italian registry, in contrast to our observations, patients treated during the pandemic were older than those treated in the corresponding period in 2019. ${ }^{5}$ It is possible that fear of infection was greater among the elderly Polish patients. Moreover, elderly patients more often experience atypical symptoms, which, combined with the symptoms of COVID-19 (dyspnea, chest discomfort), could be confusing for those patients and discourage them from contacting the healthcare services. Data available both in Poland and across the world suggest that a greater reduction in the number of treated patients is observed for NSTEMI than for STEMI patients. 5,6 This may result from various factors: NSTEMI symptoms are less conclusive, and the patient group is much more heterogeneous. Moreover, NSTEMI patients are usually older, and perhaps the above-mentioned factors regarding the elderly also played a role. Additionally, the reorganization of the healthcare system might have affected the selection of patients referred for invasive diagnostics in the first place. The frequency of prehospital SCA is often related to treatment delay. Data from Italy and China suggest that time from onset of pain to treatment has become significantly longer. ${ }^{1,5}$ Data from our analysis do not confirm these observations. We have noticed no significant differences in terms of the length of transport time from other hospitals for STEMI patients (median [IQR], 316 [171-720] $\mathrm{min}$ in 2019 vs 290 [146-697] $\mathrm{min}$ in 2020) and a trend toward longer transport time for NSTEMI patients (median [IQR], 609 [298-1560] min in 2019 vs 660 [3501589] min in 2020). It cannot be ruled out, however, that some of the patients waited for contact with a healthcare professional for a longer time, which could have caused a higher frequency of prehospital SCA in 2020.

We observed significant differences in the mode of admission to a cardiology center. During the epidemic, more patients were transferred by the Emergency Medical Services (EMS; Państwowe Ratownictwo Medyczne) directly to the hospital, with a lower number of patients transferred from other hospitals $(41.43 \%$ vs $48.05 \%$, and $40.42 \%$ vs $35.53 \%$ in 2019 and 2020 , respectively). In the present analysis, it is not possible to establish the cause of this phenomenon. Perhaps the reorganization of the healthcare system and difficulties with admitting and transferring patients to and from other hospitals resulted in a higher frequency of direct transport by the EMS, especially with regard to patients diagnosed with STEMI. Additionally, a shorter time from admission to coronary angiography was observed in STEMI patients (median [IQR], 25 [11-59] min vs 21 [10-50.5] $\mathrm{min}$ in 2019 and 2020, respectively). It seems that the suspension of planned admissions and procedures could have caused a higher availability of cardiac catheterization laboratories. On the other hand, new rules of patient admission introduced during the epidemic lengthened the initial medical activities. It is possible this did not apply to STEMI patients.

We did not observe any differences in the incidence of multivessel disease or the number of percutaneous coronary interventions. Nevertheless, during the pandemic, patients were significantly less frequently referred for coronary artery bypass graft (CABG) surgery $(7.08 \%$ vs $5.77 \%$ in 2019 and 2020, respectively). The number of patients referred for CABG during initial hospitalization did not change, but the number of planned cardiosurgical procedures after hospital discharge was reduced. Similarly, as above, we may only hypothesize about the causes. It could be that during the epidemic, doctors and patients less frequently decided to carry on with the planned postdischarge treatment. It is also possible that in patients with a less advanced multivessel disease, subsequent percutaneous coronary interventions were more frequently performed during the initial hospitalization. However, we have no data that could explain this issue. Moreover, CABG is more often performed in NSTEMI patients, who were treated less often in 2020, which could have affected the total number of $C A B G$ procedures performed.

Limitations This is a retrospective analysis, therefore it involves all the inherent limitations. Data for 2019 and 2020 come from various reporting centers, which often represent different medical units. Also, we do not yet have full clinical and angiographic characteristics of the analyzed patients, which prevents accurate 
comparison of the patients' clinical profiles in the analyzed years. Additionally, the absolute number of patients treated in 2020 cannot be directly compared with data from 2019, as not all patients during this period were reported to the PL-ACS pending data analysis.

Conclusions The COVID-19 epidemic caused a reduction in the number of patients admitted to hospitals with a diagnosis of STEMI and NSTEMI. The MI patients treated during the COVID-19 pandemic were more often taken to the hospital by direct transport of the EMS. Moreover, prehospital cardiac arrest was more frequent. The time from admission to coronary angiography was shorter among the STEMI patients, and the number of patients referred for CABG was lower.

\section{ARTICLE INFORMATION}

\section{CONFLICT OF INTEREST None declared.}

OPEN ACCESS This is an Open Access article distributed under the terms of the Creative Commons Attribution-NonCommercial-NoDerivatives 4.0 International License (CC BY-NC-ND 4.0), allowing third parties to download articles and share them with others, provided the original work is properly cited, not changed in any way, distributed under the same license, and used for noncommercial purposes only. For commercial use, please contact the journal office at kardiologiapolska@ptkardio.pl.

HOW TO CITE Hawranek M, Grygier M, Bujak K, et al. Characteristics of patients from the Polish Registry of Acute Coronary Syndromes during the COVID-19 pandemic: the first report. Kardiol Pol. 2021; 79: 192-195. doi:10.33963/KP.15756

\section{REFERENCES}

1 Tam CCF, Cheung KS, Lam S, et al. Impact of coronavirus disease 2019 (COVID-19) outbreak on ST-segment-elevation myocardial infarction care in Hong Kong, China. Circ Cardiovasc Qual Outcomes. 2020; 13: e006631.

2 Metzler B, Siostrzonek P, Binder RK, et al. Decline of acute coronary syndrome admissions in Austria since the outbreak of COVID-19: the pandemic response causes cardiac collateral damage. Eur Heart J. 2020; 41: 1852-1853.

3 Rodríguez-Leor 0, Cid-Álvarez B, 0jeda S, et al. Impact of the COVID-19 pandemic on interventional cardiology activity in Spain. REC Interv Cardiol. 2020: 2: 82-89.

4 Garcia S, Albaghdadi MS, Meraj PM, et al. Reduction in ST-segment elevation cardiac catheterization laboratory activations in the United States during COVID-19 pandemic. J Am Coll Cardiol. 2020; 75: 2871-2872.

5 De Rosa S, Spaccarotella C, Basso C, et al. Reduction of hospitalization for myocardial infarction in Italy in the COVID-19 era. Eur Heart J. 2020; 41: 2083-2088.

6 Legutko J, Niewiara $Ł$, Bartuś $S$, et al. The decline of coronary angiography and percutaneous coronary intervention procedures in patients with acute myocardial infarction in Poland during the COVID-19 pandemic. Kardiol Pol. 2020; 78: 574-576.

7 Gąsior M, Gierlotka M, Tycińska A, et al. Effects of the coronavirus disease 2019 pandemic on the number of hospitalizations for myocardial infarction: regional differences. Population analysis of 7 million people. Kardiol Pol. 2020; 78: 1039-1042.

8 Poloński L, Gasior M, Gierlotka M, et al. Polish Registry of Acute Coronary Syndromes (PL-ACS). Characteristics, treatments and outcomes of patients with acute coronary syndromes in Poland. Kardiol Pol. 2007; 65: 861-872. 